\title{
MULTIVARIATE LOGISTIC DISTRIBUTIONS ${ }^{1}$
}

\author{
By Henrick J. Malik and Bovas Abraham ${ }^{2}$ \\ University of Guelph
}

In this paper a multivariate analogue of the logistic distribution is considered. We suggest two families of multivariate logistic distributions with the property that marginal distributions are of univariate form and discuss some distributional properties of the multivariate distributions.

1. Introduction. The logistic distribution with density

$$
f(x)=\frac{e^{-x}}{\left(1+e^{-x}\right)^{2}}, \quad-\infty<x<\infty,
$$

has been widely used by Berkson [2], Berkson and Hodges [3] as a model for analyzing bioassay and other experiments involving quantal response, by Pearl and Reed [8] in studies pertaining to population growth, and by Plackett [9] in connection with problems involving censored data.

Gumbel [5], [6] suggested two bivariate exponential and two bivariate logistic distributions with exponential and logistic margins respectively. Marshall and Olkin [7] derived two multivariate exponential distributions and a multivariate Weibull distribution. In this paper a multivariate analogue of the logistic distribution is considered, with the property that marginal distributions are of univariate form and discuss some distributional properties of the multivariate distributions.

2. A multivariate logistic distribution. Dubey [4] has proved that the logistic distribution defined by equation (1.1) is a compound extreme value distribution with an exponential distribution as a compounder. We use this fact to derive a $p$-variate logistic distribution.

Let $X_{1}, X_{2}, \cdots, X_{p}$, given $\alpha$, be independent random variables with the conditional distribution function

$$
\begin{aligned}
F\left(x_{k} \mid \alpha\right)=\exp \left\{-\alpha \exp \left(-x_{k}\right)\right\}, & \\
\alpha & >0,-\infty<x_{k}<\infty, k=1,2, \cdots, p,
\end{aligned}
$$

where $\alpha$ has the density function

$$
g(\alpha)=\exp (-\alpha), \quad \alpha>0 .
$$

The conditional density and moment generating functions corresponding to $F\left(x_{k} \mid \alpha\right)$ are

$$
\begin{gathered}
f\left(x_{k} \mid \alpha\right)=\alpha \exp \left(-x_{k}\right) \exp \left\{-\alpha \exp \left(-x_{k}\right)\right\}, \quad \alpha>0,-\infty<x_{k}<\infty, \\
M\left(t_{k} \mid \alpha\right)=\alpha^{t}{ }^{t} \Gamma\left(1-t_{k}\right) .
\end{gathered}
$$

Received January 1971; revised August 1972.

${ }^{1}$ Research supported by National Research Council of Canada.

${ }^{2}$ Now at the University of Wisconsin, Madison. 
The joint distribution function of $X_{1}, X_{2}, \cdots, X_{p}$ is

$$
F\left(x_{1}, \cdots, x_{p}\right)=\int_{0}^{\infty} \prod_{i=1}^{p} F\left(x_{i} \mid \alpha\right) g(\alpha) d \alpha=\left\{1+\sum_{k=1}^{p} \exp \left(-x_{k}\right)\right\}^{-1},
$$
with density function

$$
\begin{aligned}
f\left(x_{1}, \cdots, x_{p}\right)=p ! \exp \left\{-\sum_{k=1}^{p} x_{k}\right\} & {\left[1+\sum_{k=1}^{p} \exp \left(-x_{k}\right)\right]^{-(p+1)} } \\
& -\infty<x_{k}<\infty, k=1,2, \cdots, p .
\end{aligned}
$$

We define the density function (2.6) as the $p$-variate multivariate logistic density. When $p=2$ we obtain the bivariate logistic distribution obtained by Gumbel [6].

THEOREM 2.1. If the p-dimensional random variable $\left(x_{1}, \cdots, x_{p}\right)$ has the multivariate logistic distribution (2.5) then the joint marginal distribution function of $\left(X_{1}, \cdots\right.$, $\left.X_{s}\right)(s<p)$ is that of an $s$-variate logistic distribution.

Note that the conditional density $f\left(x_{1}, \cdots, x_{s} \mid x_{s+1}, \cdots, x_{p}\right)$ of $X_{1}, \cdots, X_{s}$ given $X_{s+1}=x_{s+1}, \cdots, X_{p}=x_{p}$ is not a multivariate logistic distribution.

The moment generating function of the $p$-dimensional random variable $\left(X_{1}, \cdots, X_{p}\right)$ having the $p$-variate logistic distribution is given by

$$
M\left(t_{1}, \cdots, t_{p}\right)=\int_{0}^{\infty} \prod_{i=1}^{p} M\left(t_{i} \mid \alpha\right) g(\alpha) d \alpha=\left(1+\sum_{k=1}^{p} t_{k}\right) \prod_{k=1}^{p} \Gamma\left(1-t_{k}\right) .
$$

Theorem 2.2. For $i=1,2, \cdots, p$, let $X_{n \cdot i}$ denote the largest $i$ th component in a sequence of $n$ observations on the vector $\left(X_{1}, X_{2}, \cdots, X_{p}\right)$ having a p-variate logistic distribution then $X_{n \cdot 1}, X_{n \cdot 2}, \cdots, X_{n \cdot p}$ are asymptotically independent.

Proof. The joint probability function of $X_{n \cdot 1}, X_{n \cdot 2}, \cdots, X_{n \cdot p}$ is

$$
F^{n}\left(x_{1}, x_{2}, \cdots, x_{p}\right)=\left\{1+\exp \left(-x_{1}\right)+\cdots+\exp \left(-x_{p}\right)\right\}^{-n} .
$$

It is known, Gumbel [6], that the most probable largest values $U_{n \cdot 1}, U_{n \cdot 2}, \ldots$, $U_{n \cdot p}$ in the univariate cases are $U_{n \cdot 1}=U_{n \cdot 2}=\cdots=U_{n \cdot p}=\log _{e} n$. Hence the $p$-variate probability function for the reduced largest values $x_{1}-\log _{e} n, \ldots$, $x_{p}-\log _{e} n$ is

$$
F^{n}\left(x_{1}+\log _{e} n, \cdots, x_{p}+\log _{e} n\right) .
$$

Now passing to the limit the result follows.

Theorem 2.3. For $i=1,2, \cdots, p$, let $X_{1 . i}$ denote the smallest $i$ th component in a sequence of $n$ observations on the vector $\left(X_{1}, X_{2}, \ldots, X_{p}\right)$ with a multivariate logistic distribution; then $X_{1 \cdot 1}, X_{1 \cdot 2}, \cdots, X_{1 \cdot p}$ are not asymptotically independent.

3. Multivariate logistic distribution of type II. Gumbel's [7] bivariate logistic distribution of type II may also be extended to a $p$-variate case. A $p$-variate distribution with logistic marginal cdf's $F\left(x_{1}\right), \ldots, F\left(x_{p}\right)$ may be obtained from a system

$$
\begin{aligned}
F(x)=\prod & F\left(x_{i}\right)\left[1+\sum b_{12} \bar{F}\left(s_{1}\right) \bar{F}\left(s_{2}\right)+\sum b_{123} \bar{F}\left(s_{1}\right) \bar{F}\left(s_{2}\right) \bar{F}\left(s_{3}\right)+\cdots\right. \\
& \left.+b_{123 \cdots p} \bar{F}\left(x_{1}\right) \bar{F}\left(x_{2}\right) \cdots \bar{F}\left(x_{p}\right)\right]
\end{aligned}
$$

where $\bar{F}=1-F$ and $\sum b_{12}=\sum_{i<j} b_{i j}$, etc. 
The following theorems easily follow.

Theorem 3.1. If the p-dimensional random variable $\left(X_{1}, \cdots, X_{p}\right)$ has the $p$ variate logistic distribution of type II then the joint marginal distribution function of the s-dimensional random variable $\left(X_{1}, \cdots, X_{s}\right)(s<p)$ is that of an s-variate logistic distribution of type II.

Theorem 3.2. For $i=1,2, \ldots, p$, let $X_{n \cdot i}$ denote the largest $i$ th component in a sequence of $n$ observations on the vector $\left(X_{1}, X_{2}, \cdots, X_{p}\right)$ with a p-variate logistic distribution of type II then $X_{n \cdot 1}, X_{n \cdot 2}, \cdots, X_{n \cdot p}$ are asymptotically independent.

Theorem 3.3. For $i=1,2, \ldots, p$, let $X_{1 \cdot i}$ denote the smallest $i$ th component in a sequence of $n$ observations on the vector $\left(X_{1}, X_{2}, \cdots, X_{p}\right)$ with a p-variate logistic distribution of type II then $X_{1 \cdot 1}, X_{1 \cdot 2}, \ldots, X_{1 \cdot p}$ are asymptotically independent.

Acknowledgments. The authors are very grateful to the referees for their valuable suggestions which helped us to improve the earlier version of this paper.

\section{REFERENCES}

[1] Abraham, Bovas (1970). Study of some distributional problems from a logistic distribution. M. Sc. thesis, Univ. of Guelph.

[2] Ber Kson, Joseph (1944). Application of the logistic function to bio-assay. J. Amer. Statist. Assoc. 39 357-365.

[3] Berkson, Joseph and Hodges, J. L. Jr. (1960). A minimax estimator for the logistic function. Proc. Fourth Berkeley Symp. Math. Statist. Prob. 4 77-86. Univ. of California Press.

[4] Dubey, D. S. (1969). A new derivation of the logistic distribution. Naval Res. Logist. Quart. 16 37-40.

[5] Gumbel, E. J. (1960). Bivariate exponential distributions. J. Amer. Statist. Assoc. 55 698707.

[6] Gumbel, E. J. (1961). Bivariate logistic distributions. J. Amer. Statist. Assoc. 56 335-349.

[7] Marshall, A. W. and Olkin, INGRam (1967). A multivariate exponential distribution. $J$. Amer. Statist. Assoc. 62 30-44.

[8] Pearl, R. and Reed, L. J. (1920). On the rate of growth of the population of the United States since 1790 and its mathematical representation. Proc. Nat. Acad. Sci. 6 275288.

[9] Plackett, R. L. (1959). The analysis of life test data. Ann. Math. Statist. 30 9-19.

Department of Mathematics and Statistics UNIVERSITY OF GUELPH Guelph, Ontario, CANada 